\title{
Approximate frame representations via iterated operator systems
}

\author{
Ole Christensen, Marzieh Hasannasab
}

October 9, 2019

\begin{abstract}
It is known that it is a very restrictive condition for a frame $\left\{f_{k}\right\}_{k=1}^{\infty}$ to have a representation $\left\{T^{n} \varphi\right\}_{n=0}^{\infty}$ as the orbit of a bounded operator $T$ under a single generator $\varphi \in \mathcal{H}$. In this paper we prove that, on the other hand, any frame can be approximated arbitrarily well by a suborbit $\left\{T^{\alpha(k)} \varphi\right\}_{k=1}^{\infty}$ of a bounded operator $T$. An important new aspect is that for certain important classes of frames, e.g., frames consisting of finitely supported vectors in $\ell^{2}(\mathbb{N})$, we can be completely explicit about possible choices of the operator $T$ and the powers $\alpha(k), k \in \mathbb{N}$. A similar approach carried out in $L^{2}(\mathbb{R})$ leads to an approximation of a frame using suborbits of two bounded operators. The results are illustrated with an application to Gabor frames generated by a compactly supported function. The paper is concluded with an appendix which collects general results about frame representations using multiple orbits of bounded operators.
\end{abstract}

\section{Introduction}

In the recent and very active research area dynamical sampling [1, 2, 3, 5, 9, 11, one of the key issues is to consider frames of the form $\left\{T^{n} \varphi\right\}_{n=0}^{\infty}$, where $T$ is a bounded linear operator on a given Hilbert space $\mathcal{H}$ and $\varphi \in \mathcal{H}$. Unfortunately, the class of explicitly known frames with such a representation is very sparse: except for the Riesz bases, it only contains the Carleson frames introduced in [1]. Various alternative operator representations can be found in the literature, but each of these come with its own constraints and limitations. The purpose of this paper is to show that all of these limitations disappear if we consider approximate frame representations using suborbits of a bounded operator. Indeed, we prove that any frame $\left\{f_{k}\right\}_{k=1}^{\infty}$ can be approximated arbitrarily well in a perturbation theoretic sense by a frame $\left\{T^{\alpha(k)} \varphi\right\}_{k=1}^{\infty}$, which shares key features with the given frame. Appropriate choices of the operator $T$ already exist in the literature, e.g., the hypercyclic operators; the most important new 
aspects in the current paper is that we for some important classes of frames, e.g., frames consisting of finitely supported vectors in $\ell^{2}(\mathbb{N})$, also can specify appropriate choices of the powers $\alpha(k), k \in \mathbb{N}$. A generalization to vectors that are not finitely supported but with coordinates "decaying sufficiently fast" is presented as well. We also treat the case of frames consisting of compactly supported functions in $L^{2}(\mathbb{R})$, which for technical reasons leads to an approximation using suborbits of two bounded operators rather than one. The results are illustrated with an application to Gabor frames generated by a compactly supported function. Since the idea of using multiple suborbits is not treated detailed in the existing literature, the paper is concluded with an appendix, which discusses various frame representations using multiple suborbits and the constraints under which the results are applicable.

The paper is organized as follows. In the rest of this section we set the stage by discussing the known results and limitations concerning represesentations of frames as an orbit of a bounded operator. The results about approximate frame representations are in Section 2 ,

The following result collects some of the results from the literature about various operator representations of frames. In the entire paper we let $\mathcal{H}$ denote an infinite-dimensional separable Hilbert space. Recall that a frame $\left\{f_{k}\right\}_{k=1}^{\infty}$ is said to have infinite excess if there exists an infinite subfamily $\left\{f_{k}\right\}_{k \in I}$ such that $\left\{f_{k}\right\}_{k \in \mathbb{N} \backslash I}$ is a frame; it has finite excess if it consists of a Riesz basis and a finite number of vectors.

Theorem 1.1 Let $\left\{f_{k}\right\}_{k=1}^{\infty}$ be a frame for $\mathcal{H}$. Then the following hold.

(i) [8, 10] Assume that $\left\{f_{k}\right\}_{k=1}^{\infty}$ is overcomplete and has a representation $\left\{f_{k}\right\}_{k=1}^{\infty}=\left\{T^{n} \varphi\right\}_{n=0}^{\infty}$ for a bounded operator $T: \mathcal{H} \rightarrow \mathcal{H}$. Then $\left\{f_{k}\right\}_{k=1}^{\infty}$ is linearly independent, has infinite excess, and $f_{k} \rightarrow 0$ as $k \rightarrow \infty$.

(ii) [16] Assume that $\left\{f_{k}\right\}_{k=1}^{\infty}$ is linearly independent. Then, given any a> 1 , there exists a bounded linear operator $T: \mathcal{H} \rightarrow \mathcal{H}$ with $\|T\|=a$ and a sequence $\{\alpha(k)\}_{k=1}^{\infty} \subset \mathbb{N}_{0}$ such that $\left\{f_{k}\right\}_{k=1}^{\infty}=\left\{T^{\alpha(k)} f_{1}\right\}_{k=1}^{\infty}$.

It is worth noticing that the conditions in Theorem 1.1(i) are only necessary but not sufficient for the availability of a representation of a frame as an orbit of a bounded operator. Various characterizations of frames with such a representation can be found in [11] and [10]. A generalization of Theorem 1.1(ii) to Banach spaces appeared in [12].

The proof of Theorem 1.1(ii) is given in terms of a procedure and is not easy to implement. Our results in Section 2 are indeed inspired by Theorem 1.1(ii) and the desire to be more explicit about the operator $T$ and the sequence $\{\alpha(k)\}_{k=1}^{\infty} \subset \mathbb{N}_{0}$. Note that the result in Theorem 1.1(ii) actually does not need the frame property. On the other hand, in the current context the linear independency is indispensable: 
Proposition 1.2 Assume that $\left\{T^{\alpha(k)} \varphi\right\}_{k=1}^{\infty}$ spans an infinite-dimensional space and that $\alpha(k) \neq \alpha(\ell)$ for $k \neq \ell$. Then $\left\{T^{\alpha(k)} \varphi\right\}_{k=1}^{\infty}$ is linearly independent.

Proof. Without loss of generality we can assume that the vectors $\left\{T^{\alpha(k)} \varphi\right\}_{k=1}^{\infty}$ are ordered such that the sequence $\{\alpha(k)\}_{k=1}^{\infty}$ is increasing. Now, if $T^{\alpha(N)} \varphi \in$ $\operatorname{span}\left\{T^{\alpha(k)} \varphi\right\}_{k=1}^{N-1}$ for some $N \geq 2$, then the vector space

$$
V:=\overline{\operatorname{span}}\left\{\varphi, T \varphi, \ldots, T^{\alpha(N)-1} \varphi\right\}
$$

is invariant under the operator $T$ and hence $\left\{T^{\alpha(k)} \varphi\right\}_{k=1}^{\infty}$ only spans a finitedimensional space.

Note that Theorem 1.1(i) implies that the standard frames in harmonic analysis, e.g., overcomplete frames of translates, Gabor frames and wavelet frames, do not have representations as a (full) orbit of a bounded operator. However, by Theorem 1.1(ii) a representation as a suborbit of a bounded operator is available under the condition of linear independency, a condition that is satisfied for all regular frames of translates, Gabor frames along lattices [17], and a large class of wavelet frames [4].

\section{Approximate frame representations}

The existing literature on dynamical sampling deals with exact frame representations. The price to pay for this is that although the class of frames that can be represented as an orbit of a bounded operator is quite large [10], only few explicitly given frames of that form are known [1, 8. In this section we show that all constraints disappear if we consider approximate frame representations. Indeed, we prove that any frame can be approximated up to any desired precision using a frame of the form $\left\{T^{\alpha(k)} \varphi\right\}_{k=1}^{\infty}$ for certain choices of the operator $T$, the generator $\varphi \in \mathcal{H}$, and the powers $\alpha(k) \in \mathbb{N}$. The "approximation quality" is measured in terms of classical conditions from perturbation theory for frames, which are known to preserve key properties of the frame, e.g., the excess. For the frames of particular relevance in applications, e.g., frames consisting of finitely supported vectors in $\ell^{2}(\mathbb{N})$ and compactly supported functions in $L^{2}(\mathbb{R})$, we can specify exact choices of the powers $\alpha(k)$ for certain choices of the operator $T$ and the generator $\varphi$. The general perturbation theoretic approach is outlined in Section [2.1, and the applications to frames in $\ell^{2}(\mathbb{N})$ and $L^{2}(\mathbb{R})$ are given in Sections 2.22 .3 ,

\subsection{Perturbation theory and hypercyclic operators}

For later convenience we formulate the definition below for a frame with an arbitrary countable index set $I$. 
Definition 2.1 Let $\left\{f_{k}\right\}_{k \in I}$ be a frame for $\mathcal{H}$. Given any $\epsilon>0$, a sequence $\left\{\widetilde{f}_{k}\right\}_{k \in I} \subset \mathcal{H}$ is called an $\epsilon$-approximation of $\left\{f_{k}\right\}_{k \in I}$ if

$$
\left\|\sum c_{k}\left(f_{k}-\widetilde{f}_{k}\right)\right\|^{2} \leq \epsilon \sum\left|c_{k}\right|^{2}
$$

for all finite sequences $\left\{c_{k}\right\}$.

For sufficiently small values of $\epsilon$, an $\epsilon$-approximation $\left\{\widetilde{f}_{k}\right\}_{k \in I}$ of a frame $\left\{f_{k}\right\}_{k \in I}$ is itself a frame and shares several key properties of the frame, e.g., its excess. Furthermore, the synthesis operator and the frame operator for $\left\{f_{k}\right\}_{k \in I}$ are approximated "well" by the corresponding operators for $\left\{\widetilde{f}_{k}\right\}_{k \in I}$ :

Theorem 2.2 Consider a frame $\left\{f_{k}\right\}_{k \in I}$ for $\mathcal{H}$ with frame bounds $A, B$, and assume that $\left\{\widetilde{f}_{k}\right\}_{k \in I} \subset \mathcal{H}$ is an $\epsilon$-approximation of $\left\{f_{k}\right\}_{k \in I}$ for some $\left.\epsilon \in\right] 0, A[$. Then the following hold:

(i) $\left\{\widetilde{f}_{k}\right\}_{k \in I}$ is a frame with bounds $A\left(1-\sqrt{\frac{\epsilon}{A}}\right)^{2}$ and $B\left(1+\sqrt{\frac{\epsilon}{B}}\right)^{2}$, with the same excess as $\left\{f_{k}\right\}_{k \in I}$.

(ii) Denoting the synthesis operators and frame operators of $\left\{f_{k}\right\}_{k \in I}$ and $\left\{\widetilde{f}_{k}\right\}_{k \in I}$ by $U, \widetilde{U}$, respectively, $S, \widetilde{S}$, we have

$$
\|U-\widetilde{U}\| \leq \sqrt{\epsilon}, \quad\|S-\widetilde{S}\| \leq \sqrt{\epsilon B}\left(2+\sqrt{\frac{\epsilon}{B}}\right),
$$

and

$$
\left\|S^{-1}-\widetilde{S}^{-1}\right\| \leq \frac{\sqrt{\epsilon B}\left(2+\sqrt{\frac{\epsilon}{B}}\right)}{A^{2}\left(1-\sqrt{\frac{\epsilon}{A}}\right)^{2}} .
$$

Proof. (i) follows from Corollary 22.1.5 in [7]. For the proof of (ii), letting $\left\{c_{k}\right\}_{k=1}^{\infty} \in \ell^{2}(\mathbb{N})$,

$$
\begin{aligned}
\left\|U\left\{c_{k}\right\}_{k=1}^{\infty}-\widetilde{U}\left\{c_{k}\right\}_{k=1}^{\infty}\right\|^{2} & =\left\|\sum_{k=1}^{\infty} c_{k}\left(f_{k}-\widetilde{f}_{k}\right)\right\|^{2} \\
& \leq \epsilon \sum_{k=1}^{\infty}\left|c_{k}\right|^{2}
\end{aligned}
$$

So $\|U-\widetilde{U}\| \leq \sqrt{\epsilon}$. By the definition of frame operator, we have

$$
S-\widetilde{S}=U U^{*}-\widetilde{U} \widetilde{U}^{*}=(U-\widetilde{U}) U^{*}+\widetilde{U}\left(U^{*}-\widetilde{U}^{*}\right) .
$$

It is well known that if $B$ is any upper frame bound for a frame with synthesis operator $U$, then $\|U\| \leq \sqrt{B}$. Thus it follows from (i) that $\|\widetilde{U}\| \leq$ $\sqrt{B\left(1+\sqrt{\frac{\epsilon}{B}}\right)^{2}}$. Therefore

$$
\|S-\widetilde{S}\| \leq \sqrt{\epsilon} \sqrt{B}+\sqrt{\epsilon} \sqrt{B\left(1+\sqrt{\frac{\epsilon}{B}}\right)^{2}}=\sqrt{\epsilon B}\left(2+\sqrt{\frac{\epsilon}{B}}\right) .
$$


Also, if $A$ is any lower frame bound for a frame with frame operator $S$, then $\left\|S^{-1}\right\| \leq A^{-1}$. For the inverse of the frame operators we therefore obtain that

$$
\left\|S^{-1}-\widetilde{S}^{-1}\right\|=\left\|S^{-1}(S-\widetilde{S}) \widetilde{S}^{-1}\right\| \leq \frac{1}{A} \sqrt{\epsilon B}\left(2+\sqrt{\frac{\epsilon}{B}}\right) \frac{1}{A\left(1-\sqrt{\frac{\epsilon}{A}}\right)^{2}},
$$

as claimed.

For the applications in Sections 2.2+2.3 it is convenient to apply the following sufficient condition for $\left\{\widetilde{f}_{k}\right\}_{k \in I}$ being an $\epsilon$-approximation of $\left\{f_{k}\right\}_{k \in I}$ :

Proposition 2.3 Let $\left\{f_{k}\right\}_{k \in I}$ be a frame for $\mathcal{H}$ with lower frame bound $A$, and assume that for some $\epsilon \in\left[0, A\left[\right.\right.$ the sequence $\left\{\widetilde{f}_{k}\right\}_{k \in I} \subset \mathcal{H}$ satisfies that

$$
\sum_{k \in I}\left\|f_{k}-\widetilde{f}_{k}\right\|^{2} \leq \epsilon
$$

Then $\left\{\widetilde{f}_{k}\right\}_{k \in I}$ is an $\epsilon$-approximation of $\left\{f_{k}\right\}_{k \in I}$ and the conclusions in Theorem 2.2 hold.

The applications in the rest of the section will be based on the following special case of Proposition 2.3:

Corollary 2.4 Let $\left\{f_{k}\right\}_{k=1}^{\infty}$ be a frame for $\mathcal{H}$ with lower frame bound A: Let $\varphi \in \mathcal{H}$ and consider a bounded operator $T: \mathcal{H} \rightarrow \mathcal{H}$. Assume that for a given $\epsilon \in] 0, A\left[\right.$, and for any $k \in \mathbb{N}$ there exists a nonnegative integer $\alpha(k) \in \mathbb{N}_{0}$ such that

$$
\left\|f_{k}-T^{\alpha(k)} \varphi\right\|^{2} \leq \frac{\epsilon}{2^{k}}
$$

Then $\left\{T^{\alpha(k)} \varphi\right\}_{k=1}^{\infty}$ is an $\epsilon$-approximation of $\left\{f_{k}\right\}_{k=1}^{\infty}$ and the conclusions in Theorem 2.g hold.

Corollary 2.4 is inspired by the theory for hypercyclic operators. Recall that a bounded operator $T: \mathcal{H} \rightarrow \mathcal{H}$ is hypercyclic if there exists a vector $\varphi \in \mathcal{H}$ such that the orbit $\left\{T^{n} \varphi\right\}_{n=0}^{\infty}$ is dense in $\mathcal{H}$; any such vector $\varphi$ is called a hypercyclic vector. We refer to, e.g., [14, 13] for more information on hypercyclic operators.

Example 2.5 Let $T: \mathcal{H} \rightarrow \mathcal{H}$ be a hypercyclic operator with hypercyclic vector $\varphi$. Then, for any frame $\left\{f_{k}\right\}_{k=1}^{\infty}$ for $\mathcal{H}$ and any given $\epsilon>0$ there exists nonnegative integers $\alpha(k), k \in \mathbb{N}$, such that (2.3) holds. Thus, for sufficiently small values of $\epsilon$, we obtain a frame $\left\{T^{\alpha(k)} \varphi\right\}_{k=1}^{\infty}$ with the same excess as the frame $\left\{f_{k}\right\}_{k=1}^{\infty}$ and approximating $\left\{f_{k}\right\}_{k=1}^{\infty}$ in the sense of Definition 2.1. 
It is worth emphasizing that in Example 2.5 the obtained frames $\left\{T^{\alpha(k)} \varphi\right\}_{k=1}^{\infty}$ are only suborbits of the orbit for the operator $T$. Indeed, the full orbit of a hypercyclic operator can never be a frame, regardless of the choice of the initial vector:

Proposition 2.6 Assume that $T: \mathcal{H} \rightarrow \mathcal{H}$ is hypercyclic. Then $\left\{T^{n} y\right\}_{n=0}^{\infty}$ and $\left\{\left(T^{*}\right)^{n} y\right\}_{n=0}^{\infty}$ are not frames for any choice of $y \in \mathcal{H}$.

Proof. Let $\varphi \in \mathcal{H}$ be a hypercyclic vector and consider any $y \in \mathcal{H} \backslash\{0\}$. Then the sequence $\left\{\left\langle y, T^{n} \varphi\right\rangle\right\}_{n=0}^{\infty}=\left\{\left\langle\left(T^{*}\right)^{n} y, \varphi\right\rangle\right\}_{n=1}^{\infty}$ is unbounded, which implies that $\left\{\left\|\left(T^{*}\right)^{n} y\right\|\right\}_{n=1}^{\infty}$ is unbounded. Thus, by Theorem 7 in [3] the sequence $\left\{T^{n} y\right\}_{n=0}^{\infty}$ is not a frame. Also, considering again any $y \neq 0$,

$$
\sum_{n=0}^{\infty}\left|\left\langle\varphi,\left(T^{*}\right)^{n} y\right\rangle\right|^{2}=\sum_{n=0}^{\infty}\left|\left\langle T^{n} \varphi, y\right\rangle\right|^{2}=\infty
$$

which implies that $\left\{\left(T^{*}\right)^{n} y\right\}_{n=0}^{\infty}$ is not a frame.

The first example of a hypercyclic operator was given by Rolewicz in [19], who showed that for any $\lambda>1$ the scaled left-shift operator $T\left(x_{1}, x_{2}, x_{3}, \ldots\right):=$ $\lambda\left(x_{2}, x_{3}, \ldots\right)$ is hypercyclic on $\ell^{2}(\mathbb{N})$. More recently a hypercyclic operator has been constructed on $L^{2}(\mathbb{R})$ :

Example 2.7 Let $w: \mathbb{R} \rightarrow \mathbb{R}$ be a continuous decreasing function such that

$$
w(x)= \begin{cases}2 & \text { if } x \leq 0 \\ 1 / 2 & \text { if } x \geq 1\end{cases}
$$

Then the multiplication operator $T: L^{2}(\mathbb{R}) \rightarrow L^{2}(\mathbb{R}), T f(x):=w(x) f(x)$ is hypercyclic, [6]. In particular, reindexing any frame for $L^{2}(\mathbb{R})$ as $\left\{f_{k}\right\}_{k=1}^{\infty}$, we can approximate the frame arbitrarily well in the sense of Definition 2.1 via an appropriately chosen suborbit $\left\{T^{\alpha(k)} \varphi\right\}_{k=1}^{\infty}$.

Example 2.7 points directly to one of the key issues of the current paper: indeed, in general we do not have direct access to the numbers $\alpha(k), k \in \mathbb{N}$, such that (2.3) holds, not even for hypercyclic operators. The purpose of the next section is to show that under very natural conditions on frames in $\ell^{2}(\mathbb{N})$ and $L^{2}(\mathbb{R})$ we can be very explicit about the choices of appropriate scalars $\alpha(k)$ for certain choices of the operator $T$.

\subsection{Approximate frame representations in $\ell^{2}(\mathbb{N})$.}

In this section we will consider a frame $\left\{f_{k}\right\}_{k=1}^{\infty}$ for $\ell^{2}(\mathbb{N})$ consisting of finitely supported vectors. In the main result we consider a particular operator $T$ and 
show how to choose a vector $\varphi \in \ell^{2}(\mathbb{N})$ and powers $\alpha(k)$ such that $(2.3)$ is satisfied. In order to avoid a too cumbersome formulation of the results we will apply the following standing assumptions throughout the section:

General setup: In the entire section, let $\left\{f_{k}\right\}_{k=1}^{\infty}$ denote a frame for $\ell^{2}(\mathbb{N})$ with frame bounds $A, B$. Fix some $\lambda>1$, and consider the scaled left/rightshift operators on $\ell^{2}(\mathbb{N})$, given by

$$
T\left(x_{1}, x_{2}, \ldots\right)=\lambda\left(x_{2}, x_{3}, \ldots\right), U\left(x_{1}, x_{2}, \ldots\right)=\lambda^{-1}\left(0, x_{1}, x_{2}, \ldots\right) .
$$

For appropriately chosen nonnegative integers $\alpha(k), k \in \mathbb{N}$, let

$$
\varphi:=\sum_{n=1}^{\infty} U^{\alpha(n)} f_{n}
$$

For the case of finitely supported vectors $\left\{f_{k}\right\}_{k=1}^{\infty}$ the following result specifies how to choose the powers $\alpha(k)$ such that the vector $\varphi$ in (2.5) is well-defined and (2.3) holds:

Theorem 2.8 Under the conditions in the general setup, assume that $\left\{f_{k}\right\}_{k=1}^{\infty}$ consists of finitely supported vectors in $\ell^{2}(\mathbb{N}) ;$ for $k \in \mathbb{N}$, let $m(k)$ denote the largest index for a nonzero coordinate in $f_{k}$. Let $\{\alpha(k)\}_{k=1}^{\infty}$ be a strictly increasing sequence of nonnegative integers such that $\alpha(1)=0$ and $\alpha(k+1)-$ $\alpha(k) \geq m(k)$ for all $k \in \mathbb{N}$. Then, for any $k \in \mathbb{N}$,

$$
\left\|f_{k}-T^{\alpha(k)} \varphi\right\|^{2} \leq \frac{B \lambda^{2}}{\lambda^{2}-1} \lambda^{-2[\alpha(k+1)-\alpha(k)]} .
$$

In particular, choosing the nonnegative integers $\alpha(k)$ such that $\alpha(1)=0$ and for a given $\epsilon \in] 0, A[$,

$$
\alpha(k+1)-\alpha(k) \geq \max \left(m(k), \frac{k \ln (2)+\ln \left(\frac{B}{\epsilon}\right)+\ln \left(\frac{\lambda^{2}}{\lambda^{2}-1}\right)}{2 \ln (\lambda)}\right),
$$

the condition (2.3) is satisfied, i.e., the conclusions in Theorem 2.2 hold.

Proof. We have supp $f_{n} \subseteq\{1, \ldots, m(n)\}$ and hence

$$
\operatorname{supp} U^{\alpha(n)} f_{n} \subseteq\{1+\alpha(n), \ldots, m(n)+\alpha(n)\} ;
$$

thus the assumption $\alpha(k+1)-\alpha(k) \geq m(k), \forall k \in \mathbb{N}$, implies that the vectors $U^{\alpha(n)} f_{n}, n \in \mathbb{N}$, are perpendicular. Using that $\{\alpha(k)\}_{k=1}^{\infty}$ is assumed to be strictly increasing and that the operator $U$ is a multiple of an isometry, it follows that

$$
\sum_{n=1}^{\infty}\left\|U^{\alpha(n)} f_{n}\right\|^{2}=\sum_{n=1}^{\infty} \lambda^{-2 \alpha(n)}\left\|f_{n}\right\|^{2} \leq B \sum_{\ell=0}^{\infty} \lambda^{-2 \ell}=\frac{\lambda^{2} B}{\lambda^{2}-1}<\infty .
$$


Thus the infinite sequence defining the vector $\varphi$ is convergent. Now, fix any $k \in \mathbb{N}$. Then $T^{\alpha(k)} U^{\alpha(k)} f_{k}=f_{k}$. Also, for any $n<k$, we have $T^{\alpha(k)} U^{\alpha(n)} f_{n}=$ $T^{\alpha(k)-\alpha(n)} f_{n} ;$ since

$$
\alpha(k)-\alpha(n) \geq \alpha(n+1)-\alpha(n) \geq m(n),
$$

it follows that $T^{\alpha(k)} U^{\alpha(n)} f_{n}=0$ for $n<k$. Thus

$$
\begin{aligned}
\left\|f_{k}-T^{\alpha(k)} \varphi\right\|^{2} & =\left\|\sum_{n=k+1}^{\infty} T^{\alpha(k)} U^{\alpha(n)} f_{n}\right\|^{2} \\
& =\left\|\sum_{n=k+1}^{\infty} U^{\alpha(n)-\alpha(k)} f_{n}\right\|^{2} \\
& =\sum_{n=k+1}^{\infty} \lambda^{-2[\alpha(n)-\alpha(k)]}\left\|f_{n}\right\|^{2} \\
& \leq B \lambda^{-2[\alpha(k+1)-\alpha(k)]} \sum_{\ell=0}^{\infty} \lambda^{-2 \ell} \\
& =\frac{B \lambda^{2}}{\lambda^{2}-1} \lambda^{-2[\alpha(k+1)-\alpha(k)]} .
\end{aligned}
$$

This proves (2.6). In order to satisfy the condition (2.3) it is now enough to put the additional constraint on $\alpha(k)$ that

$$
\frac{B \lambda^{2}}{\lambda^{2}-1} \lambda^{-2[\alpha(k+1)-\alpha(k)]} \leq \epsilon 2^{-k} ;
$$

this leads to the sufficient condition stated in (2.7).

The inequality (2.7) directly express how the powers $\alpha(k)$ are influenced by the parameter $\lambda$, the support lengths $m(k)$, and the desired precision level determined by the parameter $\epsilon$. In the next result we derive an exact expression for suitable powers $\alpha(k)$ for the special case $\lambda=\sqrt{2}$.

Corollary 2.9 In the setup of Theorem [2.8, let $\lambda=\sqrt{2}$, take an upper frame bound of the form $B=2^{N}$ for some $N \in \mathbb{N}$ and a tolerance $\epsilon=2^{-j}$ for some $j \in \mathbb{N}$. Then the following hold.

(i) Without any restriction on the support sizes $m(k)$ of the vectors $f_{k}$, the condition (2.7) is satisfied if $\alpha(1)=0$ and

$$
\alpha(k)=(k-1)\left[N+j+1+\frac{k}{2}\right]+\sum_{\ell=1}^{k-1} m(\ell), k \in \mathbb{N} \backslash\{1\} .
$$


(ii) If $m(k) \leq N+j+1+k$ for all $k \in \mathbb{N}$, the condition (2.7) is satisfied if

$$
\alpha(k)=(k-1)\left[N+j+1+\frac{k}{2}\right], k \in \mathbb{N} .
$$

Proof. For the given choice of the parameters $\lambda, B, \epsilon$, a direct calculation gives that

$$
\frac{k \ln (2)+\ln \left(\frac{B}{\epsilon}\right)+\ln \left(\frac{\lambda^{2}}{\lambda^{2}-1}\right)}{2 \ln (\lambda)}=k+N+j+1 ;
$$

thus, without any assumption on the numbers $m(k)$, the condition (2.7) is satisfied if

$$
\alpha(k+1)-\alpha(k)=k+N+j+1+m(k) .
$$

Using that $\alpha(1)=0$ this yields the formula (2.10). In case $m(k) \leq N+j+1+k$ for all $k \in \mathbb{N}$, we can discard the numbers $m(k)$ in (2.12), which then leads to the formula stated in (2.11).

Under certain decay conditions on the coordinates of the vectors $f_{k}$, we can remove the assumption of finite support in Theorem 2.8. Specifically, letting $\left\{e_{k}\right\}_{k=1}^{\infty}$ denote the canonical orthonormal basis for $\ell^{2}(\mathbb{N})$, the $j$ th coordinate of the vector $f_{k}$ is $\left\langle e_{j}, f_{k}\right\rangle$; we will assume that that there exist constants $C, \beta>0$ such that

$$
\left|\left\langle e_{j}, f_{k}\right\rangle\right| \leq C e^{-\beta|j-k|}, \forall j, k \in \mathbb{N} .
$$

Note that in the frame literature this is phrased by saying that the frame $\left\{f_{k}\right\}_{k=1}^{\infty}$ is $\beta$-exponentially localized with respect to the orthonormal basis $\left\{e_{k}\right\}_{k=1}^{\infty}$; see [15].

Theorem 2.10 Under the conditions in the general setup, assume that the frame $\left\{f_{k}\right\}_{k=1}^{\infty}$ is $\beta$-exponentially localized with respect to the canonical orthonormal basis $\left\{e_{k}\right\}_{k=1}^{\infty}$ for $\ell^{2}(\mathbb{N})$, as in (2.13). Consider an increasing sequence $\{\alpha(k)\}_{k=1}^{\infty}$ of nonnegative integers such that $\alpha(1)=0$ and $\alpha(k) \geq$ $\alpha(k-1)+k-2$ for all $k \geq 2$. Then

$$
\left\|f_{1}-T^{\alpha(1)} \varphi\right\| \leq \frac{\lambda}{\lambda-1} B^{1 / 2} \lambda^{-\alpha(2)},
$$

and, for any $k \in \mathbb{N} \backslash\{1\}$,

$$
\begin{aligned}
\left\|f_{k}-T^{\alpha(k)} \varphi\right\| \leq & \frac{\lambda}{\lambda-1} B^{1 / 2} \lambda^{-[\alpha(k+1)-\alpha(k)]} \\
& +\left(\lambda e^{-\beta}\right)^{\alpha(k)}\left(\frac{C e^{-\beta}}{\sqrt{1-e^{-2 \beta}}} \sum_{n=1}^{k-1}\left(\lambda e^{-\beta}\right)^{-\alpha(n)} e^{\beta n}\right) .
\end{aligned}
$$


In particular, assuming that $\ln (\lambda)<\beta$ and fixing any $\epsilon \in] 0, A[$ if we choose the nonnegative integers $\alpha(k)$ recursively such that $\alpha(1)=0$ and for $k \geq 1$,

$$
\begin{aligned}
& \alpha(k+1) \geq \max \left(\alpha(k)+k-1, \alpha(k)+\frac{(k / 2+1) \ln 2+\ln \frac{\lambda}{\lambda-1}+\ln \sqrt{\frac{B}{\epsilon}}}{\ln \lambda},\right. \\
& \left.\frac{(k / 2+3 / 2) \ln 2+\ln \left(\sum_{n=1}^{k}\left(\lambda e^{-\beta}\right)^{-\alpha(n)} e^{\beta n}\right)+\ln \left(\frac{C e^{-\beta}}{\sqrt{1-e^{-2 \beta}}}\right)-\ln \sqrt{\epsilon}}{\beta-\ln \lambda}\right)
\end{aligned}
$$

the condition (2.3) is satisfied, i.e., the conclusions in Theorem 2.2 hold.

Proof. We first notice that the infinite series defining $\varphi$ in (2.5) is convergent; indeed, since the sequence $\{\alpha(k)\}_{k=1}^{\infty}$ is assumed to be strictly increasing and the operator $U$ is a multiple of an isometry,

$$
\sum_{n=1}^{\infty}\left\|U^{\alpha(n)} f_{n}\right\|=\sum_{n=1}^{\infty} \lambda^{-\alpha(n)}\left\|f_{n}\right\| \leq \sqrt{B} \sum_{\ell=0}^{\infty} \lambda^{-\ell}=\frac{\lambda \sqrt{B}}{\lambda-1}<\infty .
$$

Now, using that $\alpha(1)=0$, the same calculation yields that

$$
\left\|f_{1}-T^{\alpha(1)} \varphi\right\|=\left\|\sum_{n=2}^{\infty} U^{\alpha(n)} f_{n}\right\| \leq \sum_{n=2}^{\infty} \lambda^{-\alpha(n)}\left\|f_{n}\right\| \leq \frac{\lambda}{\lambda-1} B^{1 / 2} \lambda^{-\alpha(2)},
$$

as claimed in (2.14). Considering now any $k \geq 2$ and using that $f_{k}=$ $T^{\alpha(k)} U^{\alpha(k)} f_{k}$

$$
\begin{aligned}
\left\|f_{k}-T^{\alpha(k)} \varphi\right\| & =\left\|\sum_{n=1}^{k-1} T^{\alpha(k)} U^{\alpha(n)} f_{n}+\sum_{n=k+1}^{\infty} T^{\alpha(k)} U^{\alpha(n)} f_{n}\right\| \\
& \leq \sum_{n=1}^{k-1}\left\|T^{\alpha(k)-\alpha(n)} f_{n}\right\|+\sum_{n=k+1}^{\infty}\left\|U^{\alpha(n)-\alpha(k)} f_{n}\right\| .
\end{aligned}
$$

We now consider the two terms in (2.17) separately. For the first first term, note that since $\alpha(k)>\alpha(n)$ for $n=1, \ldots, k-1$, the operator $T^{\alpha(k)-\alpha(n)}$ removes the first $\alpha(k)-\alpha(n)$ coordinates of the vector the operator is acting on, and multiplies the resulting vector with $\lambda^{\alpha(k)-\alpha(n)}$. Thus

$$
\begin{aligned}
\left\|T^{\alpha(k)-\alpha(n)} f_{n}\right\| & =\lambda^{\alpha(k)-\alpha(n)}\left(\sum_{j=\alpha(k)-\alpha(n)+1}^{\infty}\left|\left\langle e_{j}, f_{n}\right\rangle\right|^{2}\right)^{1 / 2} \\
& \leq \lambda^{\alpha(k)-\alpha(n)}\left(\sum_{j=\alpha(k)-\alpha(n)+1}^{\infty} C^{2} e^{-2 \beta|j-n|}\right)^{1 / 2} .
\end{aligned}
$$


The assumption $\alpha(k) \geq \alpha(k-1)+k-2$ implies that $j-n \geq 0$ for all $n=1, \ldots, k-1$ and all $j \geq \alpha(k)-\alpha(n)+1$; thus, (2.18) yields that

$$
\begin{aligned}
\left\|T^{\alpha(k)-\alpha(n)} f_{n}\right\| & \leq \lambda^{\alpha(k)-\alpha(n)}\left(\sum_{j=0}^{\infty} C^{2} e^{-2 \beta(\alpha(k)-\alpha(n)+1+j-n)}\right)^{1 / 2} \\
& =\left(\lambda e^{-\beta}\right)^{\alpha(k)} \frac{C e^{-\beta}}{\sqrt{1-e^{-2 \beta}}}\left(\lambda e^{-\beta}\right)^{-\alpha(n)} e^{\beta n}
\end{aligned}
$$

Considering now the second term in (2.17),

$$
\begin{aligned}
\sum_{n=k+1}^{\infty}\left\|U^{\alpha(n)-\alpha(k)} f_{n}\right\| & =\sum_{n=k+1}^{\infty} \lambda^{-(\alpha(n)-\alpha(k))}\left\|f_{n}\right\| \\
& \leq B^{1 / 2} \lambda^{-(\alpha(k+1)-\alpha(k))} \sum_{\ell=0}^{\infty} \lambda^{-\ell} \\
& \leq \frac{\lambda}{\lambda-1} B^{1 / 2} \lambda^{-[\alpha(k+1)-\alpha(k)]} .
\end{aligned}
$$

Now (2.15) follows immediately from (2.17) using the expressions in (2.19) and (2.20).

Now, using that $\alpha(1)=0$, the inequalities (2.14) and (2.15) show that (2.3) holds if we choose $\alpha(k), k \geq 2$, such that

$$
\frac{\lambda}{\lambda-1} B^{1 / 2} \lambda^{-[\alpha(k+1)-\alpha(k)]} \leq \frac{1}{2} \sqrt{\epsilon 2^{-k}}
$$

and

$$
\left(\lambda e^{-\beta}\right)^{\alpha(k)}\left(\frac{C e^{-\beta}}{\sqrt{1-e^{-2 \beta}}} \sum_{n=1}^{k-1}\left(\lambda e^{-\beta}\right)^{-\alpha(n)} e^{\beta n}\right) \leq \frac{1}{2} \sqrt{\epsilon 2^{-k}}
$$

Direct calculations (which we skip) yield that (2.21) is satisfied if

$$
\alpha(k+1)-\alpha(k) \geq \frac{(k / 2+1) \ln 2+\ln \frac{\lambda}{\lambda-1}+\ln \sqrt{\frac{B}{\epsilon}}}{\ln \lambda}
$$

and that (2.22) holds if

$$
\alpha(k) \geq \frac{(k / 2+1) \ln 2+\ln \left(\sum_{n=1}^{k-1}\left(\lambda e^{-\beta}\right)^{-\alpha(n)} e^{\beta n}\right)+\ln \left(\frac{C e^{-\beta}}{\sqrt{1-e^{-2 \beta}}}\right)-\ln \sqrt{\epsilon}}{\beta-\ln \lambda} .
$$

This completes the proof of (2.16). 
Remark 2.11 The approach taken in this section is not restricted to the Hilbert space $\ell^{2}(\mathbb{N})$. In fact, taking any separable Hilbert space $\mathcal{H}$ and an orthonormal basis $\left\{e_{k}\right\}_{k=1}^{\infty}$, all the stated results can be formulated in the setting of the Hilbert space $\mathcal{H}$ using the operators

$T\left(\sum_{k=1}^{\infty} c_{k} e_{k}\right):=\lambda \sum_{k=1}^{\infty} c_{k+1} e_{k}, U\left(\sum_{k=1}^{\infty} c_{k} e_{k}\right):=\lambda^{-1} \sum_{k=1}^{\infty} c_{k} e_{k+1},\left\{c_{k}\right\}_{k=1}^{\infty} \in \ell^{2}(\mathbb{N})$.

Clearly, for the particular Hilbert space $\ell^{2}(\mathbb{Z})$ an indexing of the orthonormal basis as $\left\{e_{k}\right\}_{k=1}^{\infty}$ is not natural. An alternative would be to use the orthogonal decomposition

$$
\ell^{2}(\mathbb{Z})=\ell^{2}(\ldots,-2,-1,0) \oplus \ell^{2}(1,2,3, \ldots)=\ell^{2}\left(\mathbb{Z}_{-}\right) \oplus \ell^{2}\left(\mathbb{Z}_{+}\right)
$$

and then apply the above methods to each of the spaces $\ell^{2}\left(\mathbb{Z}_{-}\right)$and $\ell^{2}\left(\mathbb{Z}_{+}\right)$; that would then lead to an approximation of a given frame for $\ell^{2}(\mathbb{Z})$ using a union of two suborbits of appropriately chosen operators $T_{1}, T_{2}$. We will not go into details with such constructions here but just notice that they would follow the line of the approach we take in the next section for the Hilbert space $L^{2}(\mathbb{R})$.

\subsection{Approximate frame representations in $L^{2}(\mathbb{R})$.}

The key reason that makes the approach in Section 2.2 work is that the shift operator $T$ in (2.4) removes the first coordinate in a given vector in $\ell^{2}(\mathbb{N})$. Thus, the method can not directly be generalized to the shift operator on $L^{2}(\mathbb{R})$. For $L^{2}(\mathbb{R})$ we could alternatively apply the procedure outlined in Remark 2.11, but again the indexing of an orthonormal basis for $L^{2}(\mathbb{R})$ as $\left\{e_{k}\right\}_{k=1}^{\infty}$ might not appear natural. In this section we will outline an approach that is more similar to what we did in Section 2.2. In the entire section we will use the following setup:

General setup: Consider a frame $\left\{f_{k}\right\}_{k=1}^{\infty}$ for $L^{2}(\mathbb{R})$ consisting of compactly supported functions and with a common upper bound on the length of the support, see (2.24) below. Let $\left\{g_{k}\right\}_{k=1}^{\infty}$ denote the collections of functions from $\left\{f_{k}\right\}_{k=1}^{\infty}$ that are supported within $[0, \infty[$, and denote the remaining functions by $\left\{h_{k}\right\}_{k=1}^{\infty}$; note that it is a consequence of the frame condition that the families $\left\{g_{k}\right\}_{k=1}^{\infty}$ and $\left\{h_{k}\right\}_{k=1}^{\infty}$ indeed are infinite. Choose for $k \in \mathbb{N}$ the scalars $a(k), b(k), c(k), d(k) \in \mathbb{R}$ such that

$$
\operatorname{supp} g_{k} \subseteq[a(k), b(k)], \operatorname{supp} h_{k} \subseteq[c(k), d(k)] .
$$

Assume that

$$
L:=\sup _{k \in \mathbb{N}}(b(k)-a(k), d(k)-c(k))<\infty .
$$


Fix some $\lambda>1$, and define the following truncated and scaled translation operators on $L^{2}(\mathbb{R})$ :

$$
\begin{array}{llrl}
U_{1} f(x) & =\lambda^{-1} f(x-1), & & T_{1} f(x)=\lambda f(x+1) \chi_{[0, \infty[}(x) \\
U_{2} f(x)=\lambda^{-1} f(x+1), & & T_{2} f(x)=\lambda f(x-1) \chi_{]-\infty, L]}(x)
\end{array}
$$

Finally, choose increasing sequences $\{\alpha(k)\}_{k=1}^{\infty},\{\gamma(k)\}_{k=1}^{\infty}$ of positive numbers such that $\alpha(k+1)-\alpha(k) \geq b(k)$ and $\gamma(k+1)-\gamma(k) \geq L-c(k)$ for all $k \in \mathbb{N}$, and let

$$
\varphi_{1}:=\sum_{n=1}^{\infty} U_{1}^{\alpha(n)} g_{n}, \varphi_{2}:=\sum_{n=1}^{\infty} U_{2}^{\gamma(n)} h_{n} .
$$

The standing assumptions imply that for $n \neq \ell$ we have $U_{1}^{\alpha(n)} g_{n} \perp U_{1}^{\alpha(\ell)} g_{\ell}$ and $U_{2}^{\gamma(n)} h_{n} \perp U_{2}^{\gamma(\ell)} h_{\ell}$; thus, the same estimate as in (2.8) applies to show that the vectors $\varphi_{1}$ and $\varphi_{2}$ are well-defined. We will now show how the frame $\left\{f_{k}\right\}_{k=1}^{\infty}=\left\{g_{k}\right\}_{k=1}^{\infty} \cup\left\{h_{k}\right\}_{k=1}^{\infty}$ can be approximated using suborbits of the two operators $T_{1}$ and $T_{2}$, generated by the functions $\varphi_{1}$, respectively $\varphi_{2}$ :

Theorem 2.12 In the above setup, for all $k \in \mathbb{N}$ it holds that

$$
\left\|g_{k}-T_{1}^{\alpha(k)} \varphi_{1}\right\|^{2} \leq \frac{B \lambda^{2}}{\lambda^{2}-1} \lambda^{-2[\alpha(k+1)-\alpha(k)]}
$$

and

$$
\left\|h_{k}-T_{2}^{\gamma(k)} \varphi_{2}\right\|^{2} \leq \frac{B \lambda^{2}}{\lambda^{2}-1} \lambda^{-2[\gamma(k+1)-\gamma(k)]} .
$$

In particular, if we for some $\epsilon \in] 0, A\left[\right.$ choose the sequences $\{\alpha(k)\}_{k=1}^{\infty},\{\gamma(k)\}_{k=1}^{\infty}$ such that $\alpha(1)=\gamma(1)=0$ and for $k \in \mathbb{N}$,

$$
\alpha(k+1)-\alpha(k) \geq \max \left(b(k), \frac{k \ln (2)+\ln \left(\frac{2 B}{\epsilon}\right)+\ln \left(\frac{\lambda^{2}}{\lambda^{2}-1}\right)}{2 \ln (\lambda)}\right),
$$

and

$$
\gamma(k+1)-\gamma(k) \geq \max \left(L-c(k), \frac{k \ln (2)+\ln \left(\frac{2 B}{\epsilon}\right)+\ln \left(\frac{\lambda^{2}}{\lambda^{2}-1}\right)}{2 \ln (\lambda)}\right),
$$

then $\left\{T_{1}^{\alpha(k)} \varphi_{1}\right\}_{k=1}^{\infty} \cup\left\{T_{2}^{\gamma(k)} \varphi_{2}\right\}_{k=1}^{\infty}$ is an $\epsilon$-approximation of the frame $\left\{g_{k}\right\}_{k=1}^{\infty} \cup$ $\left\{h_{k}\right\}_{k=1}^{\infty}=\left\{f_{k}\right\}_{k=1}^{\infty}$ and Theorem 2.2 applies. 
Proof. The proof follows the lines of the proof of Theorem 2.8, so we only sketch it. We have already argued that the vectors $\varphi_{1}$ and $\varphi_{2}$ are well-defined. Now, fixing $k \in \mathbb{N}$, we have $T_{1}^{\alpha(k)} U_{1}^{\alpha(k)} g_{k}=g_{k}$. Also, for $n<k, \alpha(k)-\alpha(n) \geq$ $\alpha(n+1)-\alpha(n) \geq b(n)$; it follows that then $T_{1}^{\alpha(k)} U_{1}^{\alpha(n)} g_{n}=T_{1}^{\alpha(k)-\alpha(n)} g_{n}=0$. Now exactly the same argument as in (2.9) yields the conclusion in (2.28). The proof of (2.31) is similar.

We will now show explicitly how Theorem 2.12 can be applied to Gabor frames. Recall that a Gabor frame is a frame for $L^{2}(\mathbb{R})$ having the form $\left\{E_{m b} T_{n a} g\right\}_{m, n \in \mathbb{Z}}:=\left\{e^{2 \pi i b x} g(x-n a)\right\}_{m, n \in \mathbb{Z}}$ for a fixed choice of a function $g \in L^{2}(\mathbb{R})$ and some $a, b>0$. In order to apply Theorem 2.12 we only need to assume that $g$ is compactly supported. Furthermore, for special choices of the involved parameters we can be completely explicit about how to choose suitable powers $\alpha(k)$ and $\gamma(k)$ in Theorem 2.12 :

Example 2.13 Consider a Gabor frame $\left\{E_{m b} T_{n a} g\right\}_{m, n \in \mathbb{Z}}$ and assume that supp $g \subseteq[0, C]$ for some $C \in \mathbb{R}$. Choose the sequence $\left\{g_{k}\right\}_{k=1}^{\infty}$ to consist of only the positive translates $\left\{E_{m b} T_{n a} g\right\}_{m \in \mathbb{Z}, n \in \mathbb{N}_{0}}$. Furthermore, we order $\left\{g_{k}\right\}_{k=1}^{\infty}$ such that $g_{1}:=g$ and, in general, if $g_{k}=E_{m^{\prime} b} T_{n^{\prime} a} g$ for some $m^{\prime} \in \mathbb{Z}, n^{\prime} \in \mathbb{N}_{0}$, then $g_{k+1}$ corresponds to one of the following functions:

$$
E_{\left(m^{\prime} \pm 1\right) b} T_{n^{\prime} a} g, \quad E_{m^{\prime} b} T_{\left(n^{\prime} \pm 1\right) a} g .
$$

In other words, the ordering is chosen such that going from any $g_{k}$ to $g_{k+1}$, the translation parameter changes by at most $a$. Thus, we have $\operatorname{supp} g_{k+1} \subseteq$ $\left(\operatorname{supp} g_{k}+a\right) \cup\left(\operatorname{supp} g_{k}-a\right) \cup \operatorname{supp} g_{k}$. Combined with the fact that all the functions $g_{k}$ are supported within the positive real axis, this implies that for each $k \in \mathbb{N}$ there exists a positive integer $\ell_{k} \leq k$ such that $\operatorname{supp} g_{k} \subseteq$ $\left[\left(\ell_{k}-1\right) a, C+\left(\ell_{k}-1\right) a\right]$. In a similar fashion, we let $\left\{h_{k}\right\}_{k=1}^{\infty}$ consist of the subsequence $\left\{E_{m b} T_{n a} g\right\}_{m \in \mathbb{Z}, n \in\{\ldots,-3,-2,-1\}}$, ordered such that $h_{1}=T_{-a} g$ and the translation parameters in $h_{k+1}$ and $h_{k}$ differ with at most $a$. Thus supp $h_{k} \subseteq\left[-r_{k} a, C-r_{k} a\right]$ for some $r_{k} \in \mathbb{N}$ and $r_{k} \leq k$.

Now, consider the case $\lambda=\sqrt{2}$ and choose the "tolerance" as $\epsilon=2^{-j}$ for some $j \in \mathbb{N}$. Furthermore, choose an upper frame bound of the form $B=2^{N}$ for a sufficiently large value of $N \in \mathbb{N}$. Then, let $\alpha(1)=\gamma(1)=0$ and define the sequences $\{\alpha(k)\}_{k=1}^{\infty}$ and $\{\gamma(k)\}_{k=1}^{\infty}$ recursively by

$$
\begin{aligned}
\alpha(k+1)-\alpha(k) & =C+(a+1) k-a+N+j+2, \quad k \in \mathbb{N} . \\
\gamma(k+1)-\gamma(k) & =C+(a+1) k+N+j+2, \quad k \in \mathbb{N} .
\end{aligned}
$$

Explicitly, this gives

$$
\begin{aligned}
& \alpha(k)=(k-1)\left[k \frac{(a+1)}{2}+C-a+N+j+2\right], \quad k \in \mathbb{N}, \\
& \gamma(k)=(k-1)\left[k \frac{(a+1)}{2}+C+N+j+2\right], \quad k \in \mathbb{N} .
\end{aligned}
$$


Then, defining $\varphi_{1}, \varphi_{2}$ as in (2.27). Theorem 2.12 implies that the sequence $\left\{T^{\alpha(k)} \varphi_{1}\right\}_{k=1}^{\infty} \cup\left\{T^{\gamma(k)} \varphi_{2}\right\}_{k=1}^{\infty}$ is a frame for $L^{2}(\mathbb{R})$ and it is an $\epsilon$-approximation of the frame $\left\{E_{m b} T_{n a} g\right\}_{m, n \in \mathbb{Z}}$.

\section{Appendix: Multi-generator representations}

In contrast to the rest of the paper, the approximate frame representation in Theorem 2.12 is using suborbits of two operators rather than just one. There is no detailed account of frame representations using multiple orbits in the literature; for this reason we end the paper with a short collection of results concerning frame representations using multiple orbits.

Theorem 2.14 Let $\left\{f_{k}\right\}_{k=1}^{\infty}$ be a frame, which is norm-bounded below. Then there is a finite collection of vectors from $\left\{f_{k}\right\}_{k=1}^{\infty}$, to be called $\varphi_{1}, \ldots, \varphi_{J}$, and corresponding bounded operators $T_{j}: \mathcal{H} \rightarrow \mathcal{H}$, such that

$$
\left\{f_{k}\right\}_{k=1}^{\infty}=\bigcup_{j=1}^{J}\left\{T_{j}^{n} \varphi_{j}\right\}_{n=0}^{\infty} .
$$

Note that in contrast with the single-orbit representation in Theorem 1.1(i), the representation considered in Theorem 2.14 is available for the standard frames considered in applied harmonic analysis, e.g., the frames of translates, Gabor frames, wavelet frames, and generalized shift-invariant systems generated by a finite collection of nonzero functions. Note also that while frames with positive and finite excess never have a representation as an orbit of a bounded operator, they always have a representation as a finite union of operator orbit after removal of irrelevant zero-vectors:

Corollary 2.15 Let $\left\{f_{k}\right\}_{k=1}^{\infty}$ be a frame with finite excess and assume that $f_{k} \neq 0$ for all $k \in \mathbb{N}$. Then $\left\{f_{k}\right\}_{k=1}^{\infty}$ has a representation as in (2.32).

It is natural to ask how large one has to choose the parameter $J$ in Theorem 2.14, and how $J$ is related to the redundancy and excess of the frame $\left\{f_{k}\right\}_{k=1}^{\infty}$. First, by Theorem 1.1(i) the condition of $\left\{f_{k}\right\}_{k=1}^{\infty}$ being norm-bounded below implies that for overcomplete frames we necessarily have $J \geq 2$ in Theorem 2.14. Technically, Theorem 2.14 was proved using the Feichtinger Theorem (an equivalent form of the long-standing Kadison-Singer Conjecture, which was finally confirmed in [18]) which states that every frame that is normbounded below can be represented as a finite union of Riesz sequences. Thus, it would be natural to expect that we would have to choose $J$ at least as large as the minimal number of Riesz sequences in the Feichtinger decomposition. This turns out not to be true. We will demonstrate this by exhibiting a class of frames for which we can actually take $J=2$, regardless of the number of Riesz sequences appearing in the Feichtinger decomposition. 
Example 2.16 Consider any frame that has a representation as a bi-infinite orbit $\left\{T^{n} \varphi\right\}_{n \in \mathbb{Z}}$ for a bounded and bijective operator $T: \mathcal{H} \rightarrow \mathcal{H}$. Then

$$
\left\{T^{n} \varphi\right\}_{n \in \mathbb{Z}}=\left\{T^{n} \varphi\right\}_{n=0}^{\infty} \cup\left\{\left(T^{-1}\right)^{n} T^{-1} \varphi\right\}_{n=0}^{\infty},
$$

i.e., the frame $\left\{T^{n} \varphi\right\}_{n \in \mathbb{Z}}$ can be represented as a union of two orbits. Frames with a representation as a bi-infinite orbit of a bounded operator were characterized in [9]; in particular, the assumptions are satisfied for frames of translates $\left\{T_{k b} \varphi\right\}_{k \in \mathbb{Z}}$ in $L^{2}(\mathbb{R})$. Such frames can be constructed with arbitrarily large redundancy (thus forcing a large number $J$ in the Feichtinger decomposition). Indeed, it is well known that the integer-translates $\left\{T_{k} \text { sinc }\right\}_{k \in \mathbb{Z}}$ of the sinc-function forms an ortonormal basis for the Paley-Wiener space

$$
P W:=\left\{f \in L^{2}(\mathbb{R}) \mid \operatorname{supp} \hat{f} \subseteq[-1 / 2,1 / 2]\right\} .
$$

It follows that for any $N \in \mathbb{N}$, the family $\left\{T_{k / N} \operatorname{sinc}\right\}_{k \in \mathbb{Z}}$ is an overcomplete frame for $P W$ consisting of the union of $N$ orthonormal bases. Thus the proof of Theorem 1.1 (ii) suggests that we must take $J \geq N$; however, by (2.33) the frame can be decomposed as a union of just two operator orbits.

An intermediate step between a representation of a frame as a single orbit and as in (2.32) would be to consider multiple orbits generated by the same operator $T$. The next example shows that in contrast to the case of frames represented as a single orbit, such frames are not forced to be linearly independent, and they might be norm-bounded below. The example consists of a construction of two families of vectors, each of which are complete and satisfy the Bessel condition but not the lower frame condition; however, the union of the two systems forms a frame that can be represented as a union of two operator systems, generated by the same operator $T$ but using different generators.

Example 2.17 Let $\left\{e_{k}\right\}_{k=1}^{\infty}$ denote an orthonormal basis for $\mathcal{H}$. Our purpose is to consider the sequence $\left\{e_{k}+e_{k+1}\right\}_{k=1}^{\infty} \cup\left\{e_{k}-e_{k+1}\right\}_{k=1}^{\infty}$ and show that

(i) $\left\{e_{k}+e_{k+1}\right\}_{k=1}^{\infty} \cup\left\{e_{k}-e_{k+1}\right\}_{k=1}^{\infty}$ is linearly dependent, and hence can not be represented as an orbit of a single operator (bounded or not).

(ii) $\left\{e_{k}+e_{k+1}\right\}_{k=1}^{\infty} \cup\left\{e_{k}-e_{k+1}\right\}_{k=1}^{\infty}$ is a frame and can be represented in the form $\cup_{j=1}^{2}\left\{T^{n} \varphi_{j}\right\}_{n=0}^{\infty}$ for a bounded operator $T: \mathcal{H} \rightarrow \mathcal{H}$ and some $\varphi_{1}, \varphi_{2} \in \mathcal{H}$

First, the result in (i) follows immediately from the observation that $0=$ $\left(e_{1}+e_{2}\right)-\left(e_{1}-e_{2}\right)-\left(e_{2}+e_{3}\right)-\left(e_{2}-e_{3}\right)$. Hence, the family $\left\{e_{k}+e_{k+1}\right\}_{k=1}^{\infty} \cup\left\{e_{k}-\right.$ $\left.e_{k+1}\right\}_{k=1}^{\infty}$ can not be represented as an orbit of a single operator. To prove that it can be represented as a union of two operator orbits we note that by Example 
5.4.6 in [7] the family $\left\{e_{k}+e_{k+1}\right\}_{k=1}^{\infty}$ is a complete Bessel sequence, but not a frame. Considering the bounded linear operator $T: \mathcal{H} \rightarrow \mathcal{H}$ defined by $T e_{k}:=$ $e_{k+1}, k \in \mathbb{N}$ and letting $\varphi_{1}:=e_{1}+e_{2}$, we have $\left\{e_{k}+e_{k+1}\right\}_{k=1}^{\infty}=\left\{T^{n} \varphi_{1}\right\}_{n=0}^{\infty}$. A slight modification of the argument proves that also $\left\{e_{k}-e_{k+1}\right\}_{k=1}^{\infty}$ is a complete Bessel sequence but not a frame; also, with the same operator $T$ and $\varphi_{2}:=e_{1}-e_{2}$, we have $\left\{e_{k}-e_{k+1}\right\}_{k=1}^{\infty}=\left\{T^{n} \varphi_{2}\right\}_{n=0}^{\infty}$.

To prove the frame property in (ii) it is enough to find a Bessel sequence leading to perfect reconstruction. By a direct calculation, for any $f \in \mathcal{H}$,

$$
f=\sum_{k=1}^{\infty}\left\langle f, \frac{1}{2} e_{k}\right\rangle\left(e_{k}+e_{k+1}\right)+\sum_{k=1}^{\infty}\left\langle f, \frac{1}{2} e_{k}\right\rangle\left(e_{k}-e_{k+1}\right)
$$

this proves that $\left\{e_{k}+e_{k+1}\right\}_{k=1}^{\infty} \cup\left\{e_{k}-e_{k+1}\right\}_{k=1}^{\infty}$ indeed is a frame, with dual frame $\left\{\frac{1}{2} e_{k}\right\}_{k=1}^{\infty} \cup\left\{\frac{1}{2} e_{k}\right\}_{k=1}^{\infty}$.

While certain frames with finite excess can be represented using unions of operator orbits, this possibility disappear if we insist on all orbits being generated by the same operator:

Proposition 2.18 Consider a frame $\left\{f_{k}\right\}_{k=1}^{\infty}$ with finite and strictly positive excess. Then there does not exist a bounded operator $T: \mathcal{H} \rightarrow \mathcal{H}$ and a finite number of generators $\left\{\varphi_{j}\right\}_{j=1}^{J}$ such that

$$
\left\{f_{k}\right\}_{k=1}^{\infty}=\bigcup_{j=1}^{J}\left\{T^{n} \varphi_{j}\right\}_{n=0}^{\infty} .
$$

Proof. Let $\left\{f_{k}\right\}_{k=1}^{\infty}$ be a frame for $\mathcal{H}$ with finite excess. Assume that there is an operator $T \in B(\mathcal{H})$ and $\varphi_{1}, \ldots, \varphi_{J} \subset\left\{f_{k}\right\}_{k=1}^{\infty}$ such that (2.34) holds. Since $\left\{f_{k}\right\}_{k=1}^{\infty}$ has positive excess, there exists an $\ell \in \mathbb{N}_{0}$ and $j_{0} \in\{1, \ldots, J\}$ and scalars $\left\{c_{n}^{j}\right\}_{j=1, \cdots, J, n \in \mathbb{N}_{0}} \subset \mathbb{C}$ such that

$$
T^{\ell} \varphi_{j_{0}}=\sum_{j \neq j_{0}} \sum_{n=0}^{\infty} c_{n}^{j} T^{n} \varphi_{j}+\sum_{n \neq \ell} c_{n}^{j_{0}} T^{n} \varphi_{j_{0}}
$$

Using that the frame $\left\{f_{k}\right\}_{k=1}^{\infty}$ is assumed to have finite excess, we can choose $N \in \mathbb{N}$ such that $\cup_{j=1}^{J}\left\{T^{n} \varphi_{j}\right\}_{n=N}^{\infty}$ is a Riesz sequence. Now, take any $M>N$. Then, acting with $T^{M}$ yields

$$
\begin{aligned}
T^{M+\ell} \varphi_{j_{0}} & =\sum_{j \neq j_{0}} \sum_{n=0}^{\infty} c_{n}^{j} T^{n+M} \varphi_{j}+\sum_{n \neq \ell} c_{n}^{j_{0}} T^{n+M} \varphi_{j_{0}} \\
& =\sum_{j \neq j_{0}} \sum_{n=M}^{\infty} c_{n}^{j} T^{n} \varphi_{j}+\sum_{n \geq M, n \neq M+\ell} c_{n}^{j_{0}} T^{n} \varphi_{j_{0}} .
\end{aligned}
$$


This implies that

$$
T^{M+\ell} \varphi_{j_{0}} \in \overline{\operatorname{span}}\left\{T^{n} \varphi_{j}\right\}_{n \geq M, j \neq j_{0}} \cup\left\{T^{n} \varphi_{j_{0}}\right\}_{n \geq M, n \neq \ell+M}
$$

contradicting that $\cup_{j=1}^{J}\left\{T^{n} \varphi_{j}\right\}_{n=N}^{\infty}$ is a Riesz basis.

Acknowledgment: The authors would like to thank the anonymous referees for their helpful comments which improved the presentation of the results.

\section{References}

[1] Aldroubi, A., Cabrelli, C., Molter, U., and Tang, S.: Dynamical sampling. Appl. Harm. Anal. Appl. 42 (2017), 378-401.

[2] Aldroubi, A., Cabrelli, C., Çakmak, A. F., Molter, U., and Petrosyan, A.: Iterative actions of normal operators. J. Funct. Anal. 272 no. 3 (2017), 1121-1146.

[3] Aldroubi, A. and Petrosyan, A.: Dynamical sampling and systems from iterative actions of operators, Frames and other bases in abstract and function spaces, 1526, Appl. Numer. Harmon. Anal., Birkhuser/Springer, Cham, 2017.

[4] Bownik, M., and Speegle, D.: Linear independence of Parseval wavelets. Illinois J. Math. 54 (2010), 771-785.

[5] Cabrelli, C., Molter, U, Paternostro, V., and Philipp, F.: Dynamical sampling on finite index sets. Preprint, 2017.

[6] Chen, K. Y.: On aperiodicity and hypercyclic weighted translation operators. J. Math. Anal. Appl. 462 (2018), no. 2, 1669-1678

[7] Christensen, O.: An introduction to frames and Riesz bases. Second expanded edition. Birkhäuser (2016)

[8] Christensen, O. and Hasannasab, M.: Frame properties of systems arising via iterative actions of operators Appl. Comp. Harm. Anal. 46 (2019), 664-673.

[9] Christensen, O., and Hasannasab, M.: Operator representations of frames: boundedness, duality, and stability Integral Equations an Operator Theory 88 (2017), no. 4, 483-499.

[10] Christensen, O., Hasannasab, M., and Philipp, F.: Frame Properties of Operator Orbits. Accepted for publication in Math. Nach., 2019 
[11] Christensen, O., Hasannasab, M., and Rashidi, E.: Dynamical sampling and frame representations with bounded operators. J. Math. Anal. Appl. 463 no. 2 (2018), 634-644.

[12] Grivaux, S.: Construction of operators with prescribed behaviour, Arch. Math. (Basel), 81 (2003), no. 3, 291-299.

[13] Grosse-Erdmann K. G.: Universal families and hypercyclic operators Bulletin of the American Mathematical Society. 1999;36(3):345-81.

[14] Grosse-Erdmann, K. G., and Manguillot, A. P.: Linear chaos, Springer Science \& Business Media, (2011).

[15] Gröchenig, K.: Localization of frames, Banach frames, and the invertibility of the frame operator. J. Fourier Anal. Appl. 10 (2004), 105-132.

[16] Halperin, I., Kitai, C., and Rosenthal, P.: On orbits of linear operators. Journal of the London Mathematical Society 2, 3 (1985), 561-565.

[17] Linnell, P.: Von Neumann algebras and linear independence of translates. Proc. Amer. Math. Soc. 127 no. 11 (1999), 3269-3277.

[18] Marcus, A. W., Spielman, D. A., and Srivastava, N.: Interlacing families II: Mixed characteristic polynomials and the Kadison-Singer problem. Ann. of Math. (2) 182 no. 1, (2015), 327-350.

[19] Rolewicz, S.: On orbits of elements. Studia Mathematica 32, 1 (1969), $17-22$.

Ole Christensen

Technical University of Denmark

DTU Compute

Building 303, 2800 Lyngby

Denmark

Email: ochr@dtu.dk
Marzieh Hasannasab

Technical University of Kaiserslautern

Paul-Ehrlich Straße Gebäude 31

67663 Kaiserslautern

Germany

hasannas@mathematik.uni-kl.de 